\title{
PERIODIC CONTROL OF RATE OF DRUG PERMEATION THROUGH THE SKIN WITH IONTOPHORESIS
}

\author{
AKIHIRo YAMASHITA, KoHJI TANAKA \\ AND KaKUJi TOJO \\ Department of Biochemical Engineering and Science, College of \\ Computer Science and Systems Engineering, Kyushu Institute of \\ Technology, Fukuoka 820
}

\begin{abstract}
Key Words: $\quad$ Drug Delivery System, Transdermal Therapeutic System, Electric Field, Iontophoresis, Benzoic Acid
\end{abstract}

\begin{abstract}
Iontophoresis, which enhances the rate of charged drugs through a membrane by applying an electric current, is a novel promising technique for a drug delivery system. The authors used iontophoresis to enhance the skin permeability of drugs in transdermal delivery. In vitro permeation experiments across a piece of excised hairless mouse skin were made with/without electric field application. A model drug, benzoic acid (BA, MW=122), did not passively penetrate through the intact hairless mouse skin at $\mathrm{pH}=7.4$ (almost completely ionized); however, it could appreciably permeate through the skin when an electric current density of $3.0 \mathrm{~A} / \mathrm{m}^{2}$ was applied. The permeation flux of BA through the skin was linearly changed with the electric current density, which makes it possible to control drug concentration in the blood periodically. The permeation flux of dexamethasone sodium $\mathrm{m}$-sulfobenzoate $(\mathrm{MW}=599)$, which is larger than $\mathrm{BA}$, was also enhanced by applying iontophoresis.

Computer simulation was performed for iontophoresis experiments using transport parameters determined from passive diffusion experiments. Experimental findings under iontophoresis were well correlated by the results of computer simulation, which may be useful for designing an optimal administrating schedule for a specific drug.
\end{abstract}

\section{Introduction}

The transdermal therapeutic system (TTS), or drug absorption through the skin into the human body is a promising drug delivery system (DDS). One of the difficult problems in TTS is to overcome mass transfer resistance of the skin. The stratum corneum, the outermost layer of the skin, is hydrophobic which prevents drugs and toxic substances from penetrating the human body. Iontophoresis is a novel technique for enhancing the rate of ionic drug transport through the membrane due to the electrical potential gradient as well as possible convective flow across the skin caused by the electric fields. We used the iontophoretic technique for TTS, choosing benzoic acid and dexamethasone sodium msulfobenzoate as model ionic drugs. Since the electric current can be easily controlled by changing its intensity or even by switching on and off periodically, the rate of mass transfer can also be changed with it. We also observed how the permeation rate changed with the on-off operation of the electric current during the course of experiments.

* Received on May 5, 1996. Correspondence concerning this article should be addressed to A. Yamashita.

Presented at the 60th Annual Meeting of The Society of Chemical Engineers, Japan, at Osaka, March 1995.

\section{Theoretical}

The steady-state mass flux of a solute of interest across a uniform membrane may be approximated as follows (Crank, 1975):

$$
\left(\frac{d Q_{t}}{d t}\right)_{S S} \approx \frac{K D C_{D}}{L}
$$

where $Q_{t}$ is the cumulative amount of solute of interest permeated through the membrane till time $t$ per unit membrane area $\left[\mathrm{kg} / \mathrm{m}^{2}\right],\left(d Q_{t} / d t\right)_{s S}$ is the steady state mass flux $\left[\mathrm{kgs}^{-1} \mathrm{~m}^{-2}\right], C_{D}$ is the concentration of solute of interest in the donor solution $\left[\mathrm{kg} / \mathrm{m}^{3}\right], D$ is the apparent diffusion coefficient of the solute of interest in the membrane $\left[\mathrm{m}^{2} / \mathrm{s}\right], K$ is the partition coefficient defined by the ratio of concentration at the surface of the membrane to that in the solution right adjacent to the membrane $\left(\approx C_{D}\right)[-]$, and $L$ is the membrane thickness [m]. The partition coefficient may be calculated from Eq. (1) by solving it for $K$, after finding the apparent diffusion coefficient by the timelag method (Barrer, 1951), assuming no solutemembrane interactions, i.e.,

$$
D=\frac{L^{2}}{6 t_{d}}
$$


where $t_{d}$ is the time lag for permeation [s].

The skin, however, may be divided into two layers, i.e., thin surface layer called the stratum corneum and the other viable epidermis (Tojo, Chiang, 1987). The stratum corneum is mostly composed of lipids and dead cells and is highly hydrophobic, whereas the viable epidermis is of hydrophilic living cells with various enzymes. A detailed description of the mathematical system which includes both diffusion and possible bulk flow through the skin (water shift), considering also partitioning between two solid layers, can be found in the literature (Tojo, 1987, Tojo, 1989). The rate of drug transfer can be explained by the authors' twolayer mathematical model. After determining permeation parameters (diffusion coefficients and partition coefficients for the stratum corneum as well as the viable epidermis) from experimental data by the twolayer model, a computer simulation was made with PC-SKIN II, which is based on the same two-layer model, to deal with drug transport by diffusion and by convection across the skin.

\section{Materials and Method}

\subsection{Preparation}

Benzoic acid (BA, MW=122, $\mathrm{pKa}=4.2$, Wako Pure Chemical Co., Japan) was used as a model ionic substance. Phosphate buffer solutions with various pH were prepared for experimental solutions which changed the degree of ionization of BA. The pH's were 7.4 (BA: almost completely ionized), 6.0 (95\% ionized), 5.0 ( $72 \%$ ionized), and 4.0 (35\% ionized).

In drug permeation experiments, a $(0.03 \mathrm{~m} \times$ $0.03 \mathrm{~m}$ ) piece of intact or tape-stripped skin was excised from a hairless mouse (Kyudo Co., Japan). Tape stripping was carried out 20 times with adhesive tape (Cellophane tape ${ }^{\circledR}$, Nichiban Co., Japan) so as to remove the stratum corneum completely from the surface of the skin (Tojo, 1988). Either the intact or tape-stripped skin was placed between the donor and receptor compartments of a diffusion chamber (Permcell ${ }^{\circledR}$, Fig. 1, Kokura Glass Co., Japan, effective surface area $=6.0 \times 10^{-5} \mathrm{~m}^{2}$, effective cell volume $=$ $\left.5.0 \times 10^{-6} \mathrm{~m}^{3} \mathrm{each}\right)$. Saturated BA solution with some BA crystals to maintain unit thermodynamic activity was put in the donor compartment, while the pure buffer solution of $\mathrm{pH}$ of 7.4 was put in the receptor compartment, mimicking human physiological conditions.

\subsection{Permeation experiments}

Iontophoresis experiments were performed at a constant electric current using a Model 6911 (Metronix Inc., Tokyo, Japan). Multiple samples of $1.0 \times 10^{-7} \mathrm{~m}^{3}$ were taken from the receptor solution at various time intervals, and the same amount of pure buffer solution was added into the receptor to keep the

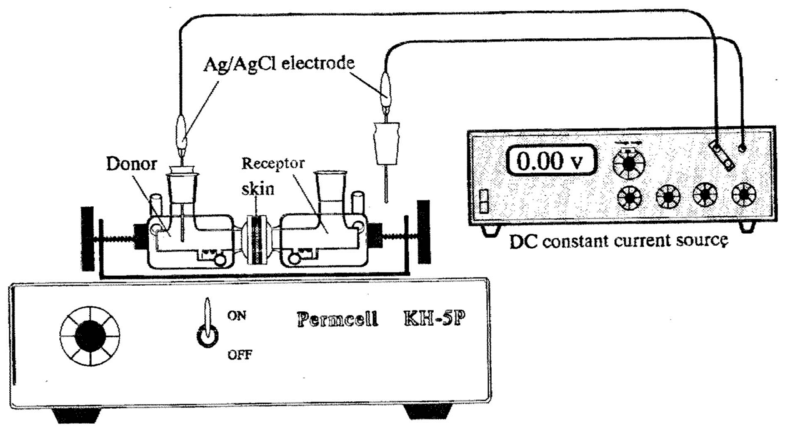

Fig. 1 Experimental setup for studying in vitro skin permeation by iontophoresis



Fig. 2 Time course of cumulative amount of benzoic acid permeated through intact and tape-stripped skin

volume constant during the entire period of experiment. BA concentrations of the samples were analyzed by HPLC (LC-6A System, Shimadzu Co., Japan). All the experiments were done at $310 \mathrm{~K}$.

Dexamethasone sodium m-sulfobenzoate (DMSB, MW=599, Wako Pure Chemical Co., Japan) was also used in the same manner as stated above as a possible larger molecule drug used in TTS.

\section{Results and Discussion}

Figure 2 shows the cumulative amount of BA permeated through the intact and tape-stripped skin at both the donor and receptor $\mathrm{pH}$ of 7.4. Clearly BA hardly penetrate through the intact skin, while it diffused across the tape-stripped skin. Because BA was almost completely ionized at this $\mathrm{pH}$, it acted as a "water-soluble" substance. The stratum corneum is hydrophobic and that made it difficult for BA to diffuse into the skin. BA could, however, permeate through the tape-stripped viable skin because the stripped skin or the viable epidermis has fairly high hydrophilicity. Determined diffusion coefficients and partition coefficients are tabulated in Table 1 .

Figure 3 shows the result of the cumulative 


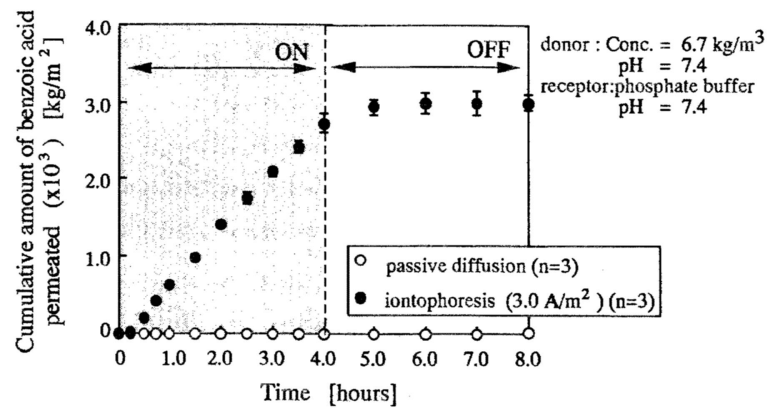

Fig. 3 Time course of cumulative amount of benzoic acid permeated through the intact skin with donor $\mathrm{pH}$ of 7.4

amount of BA permeated through the intact skin at both the donor and receptor $\mathrm{pH}$ of 7.4 with/without electric current of $3.0 \mathrm{~A} / \mathrm{m}^{2}$ application. The figure shows that although BA could not passively diffuse through the intact skin, application of electric current made it possible for BA to permeate the skin. Figures 4-6 show results of the effect of various donor pH's, i.e., $\mathrm{pH}$ of $6.0,5.0$, and 4.0 on the permeation profiles under iontophoresis. As donor $\mathrm{pH}$ decreased, the rate of passive diffusion through the skin increased and the difference between the permeation fluxes with and without electric current decreased. The lower the $\mathrm{pH}$, the lower the degree of ionization is and it reaches as low as approximately $35 \%$ when $\mathrm{pH}$ of 4.0 is achieved. As an organic compound, BA could dissolve more easily into the hydrophobic skin surface (stratum corneum) as the donor $\mathrm{pH}$ decreases, which made the steady state permeation flux higher in accordance with Eq. (1) because a larger $K$ value may be expected. Although the intact skin is somehow protective to an acidic solution, it could be damaged when exposed to a very acidic (e.g. $\mathrm{pH}=4.0$ ) solution for a long period of time. That might also help the permeation flux increased.

The relationship between steady state permeation flux and $\mathrm{pH}$ of the donor solution is shown in Fig. 7. It also includes a relationship between the ratio of molecular form of BA calculated from a Henderson-Hasselbalch type equation

$$
\mathrm{pH}=\mathrm{pK}_{\mathrm{a}}+\log _{10}\left(\frac{\left[\mathrm{C}_{6} \mathrm{H}_{5}-\mathrm{COO}^{-}\right]}{\left[\mathrm{C}_{6} \mathrm{H}_{5}-\mathrm{COOH}\right]}\right)
$$

with $\mathrm{pH}$ of the donor solution, which summarizes the above discussion. BA did not passively diffuse through the intact skin for a $\mathrm{pH}$ greater than 6.0 because almost all the BA molecules should be ionized. However, once electric current was applied, BA could partition in the intact skin and could permeate through it. As the donor $\mathrm{pH}$ decreased, the ratio of iontophoretic to passive flux one decreased, although absolute values of both passive and

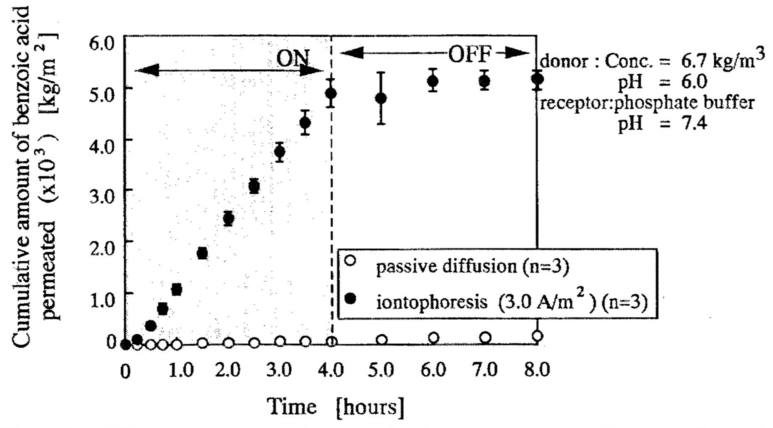

Fig. 4 Time course of cumulative amount of benzoic acid permeated through the intact skin with donor $\mathrm{pH}$ of 6.0

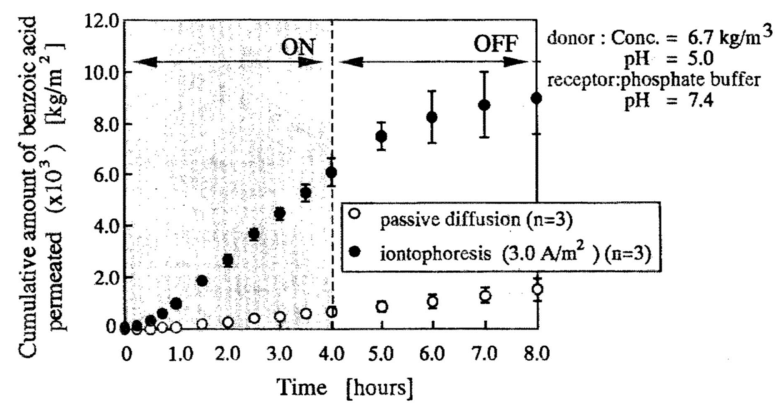

Fig. 5 Time course of cumulative amount of benzoic acid permeated through the intact skin with donor $\mathrm{pH}$ of 5.0

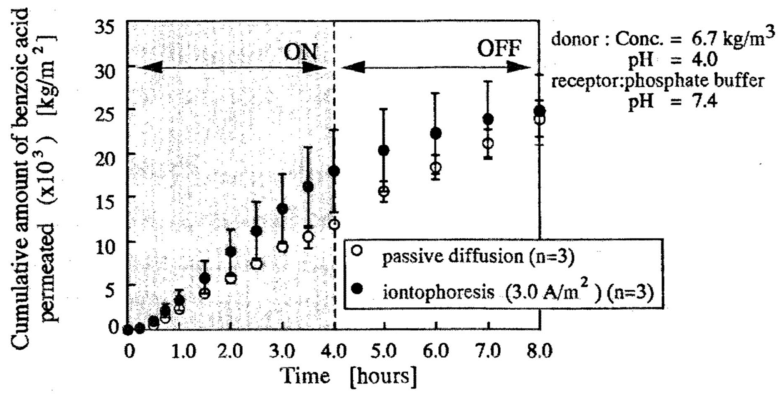

Fig. 6 Time course of cumulative amount of benzoic acid permeated through the intact skin with donor $\mathrm{pH}$ of 4.0

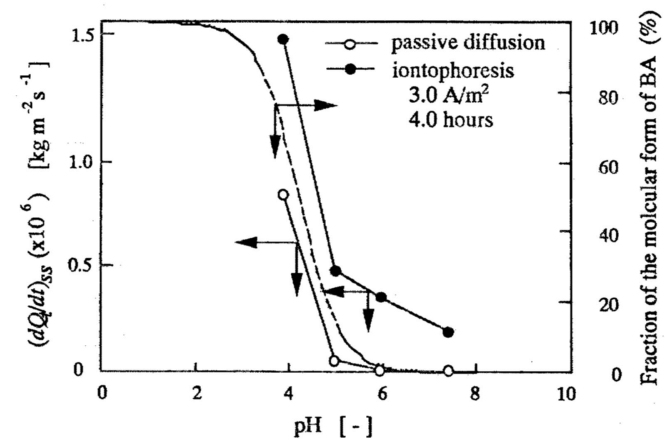

Fig. 7 Relationship between steady state flux of BA permeation and $\mathrm{pH}$ of donor solution 


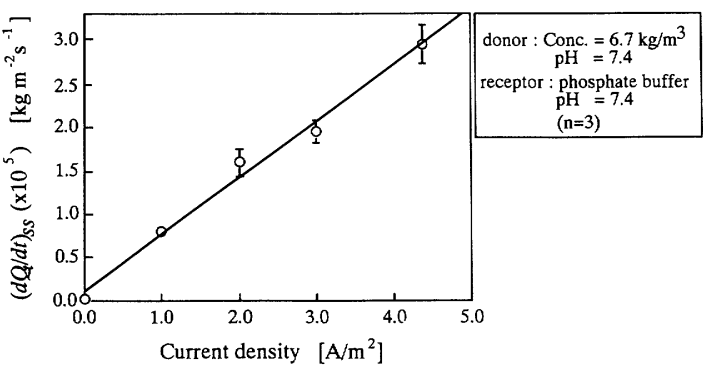

Fig. 8 Relationship between current density and steady state permeation flux of BA at donor $\mathrm{pH}$ of 7.4

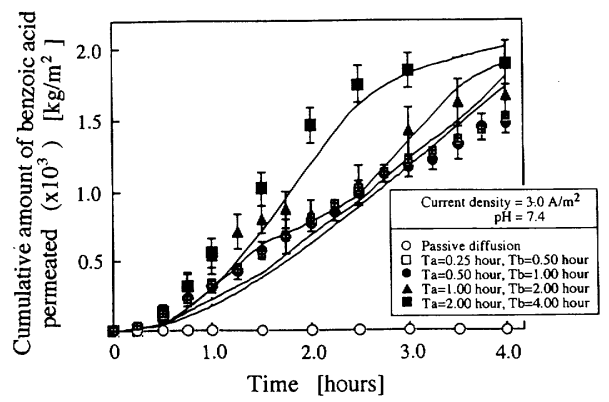

Fig. 9 Time course of cumulative amount of benzoic acid permeated through intact skin with various periodical application of electric current at donor $\mathrm{pH}$ of 7.4

iontophoretic permeation fluxes increased. This finding may suggest that iontophoresis is more crucial for drugs that cannot passively diffuse across the intact skin.

A linear relationship was observed between the steady-state permeation flux for BA and the current density (Fig. 8). Figure 8 implies that the permeation flux of a drug may be controlled by changing the current density or by simply changing the intensity of the electric current, which could be helpful for precise control of a drug concentration in plasma when applied clinically.

Figure 9 represents the cumulative amount of BA permeated with the electric current periodically switched on and off during the course of experiment. $T_{a}$ in the figure refers to an application duration of time, whereas $T_{b}$ is the entire period which includes both on-time and off-time. Solid curves are those calculated by the mathematical model (Tojo, 1989). Data showed that the periodic on and off of electric current could change the drug permeation flux drastically. Moreover, the theoretical calculations were well correlated with the experimental data, which may be useful for designing an optimal administrating schedule for a specific drug.

Figure 10 is a result of computer simulation of plasma concentration of a "BA-like" drug under

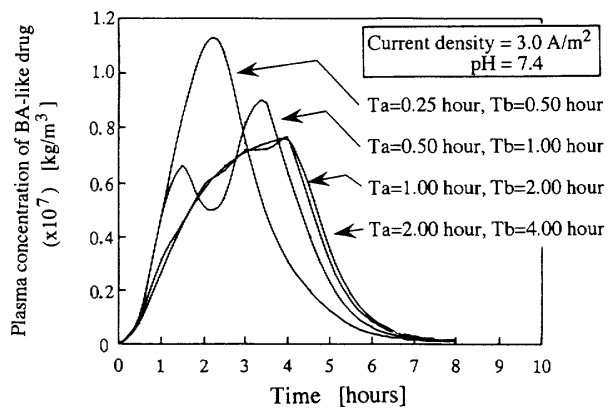

Fig. 10 Computer simulation of plasma concentration profile for a "BA-like" drug under various modes of application

various patterns of clinical application of iontophoresis. In this simulation, iontophoretic enhancement of drug transport is taken into account in addition to passive diffusion due to concentration gradient as well as possible bulk flow across the skin due to osmotic gradient. Data used for this simulation are tabulated in Table 1. No electric current was applied beyond four hours after the treatment. Since the half-life for the drug in the body is assumed to be small $(1800 \mathrm{~s})$, and since the skin penetration reaches steady state with a short time lag, the plasma concentration level can be easily changed by switching on and off the electric current. This enables the chronopharmacological control of drug concentration in plasma.

Iontophoretic permeation results of DMSB is shown in Fig. 11 with two different application patterns in addition to the passive diffusion. Applications were made for the first twelve hours with two different patterns. As for BA, DMSB was hardly found in the receptor solution up to half a day without applying electric current (passive diffusion). Although DMSB has a molecular weight of almost 600 , the rate of permeation could be drastically enhanced by the application of electric current. The rate may also be changed by switching on and off periodically and the cumulative amount of DMSB permeated may be different, even if total application time is the same. Therefore designing application patterns of electric current may be important when one needs to control the drug concentration in plasma chrono-pharmacologically.

\section{Conclusion}

1. Benzoic acid (BA) ionized completely at $\mathrm{pH}$ of 7.4 hardly permeates through the intact skin but the tape-stripped skin.

2. The permeation flux of BA through the intact skin can be greatly enhanced by applying electric 
Table 1 Determined and used parameters for computer simulation

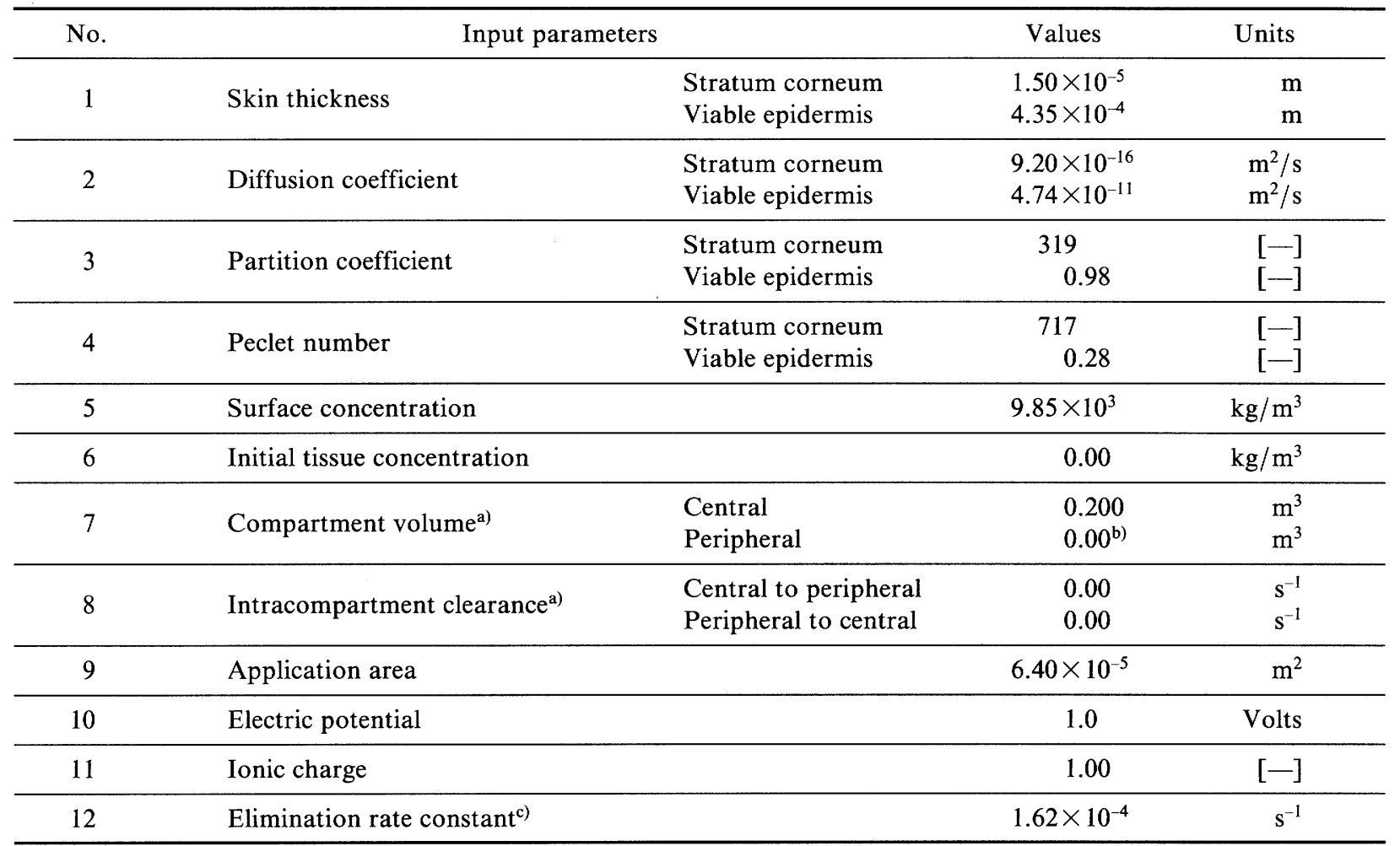

a) One-Compartment model is assumed.

b) Value of any number but " 0.00 " may be accepted for actual calculation.

c) Value was estimated from its MW.

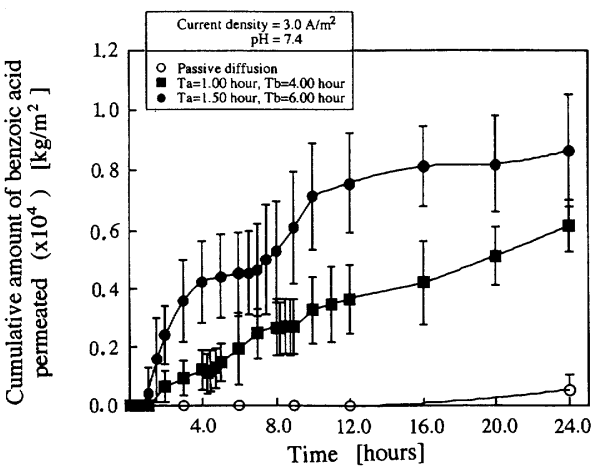

Fig. 11 Time course of cumulative amount of dexamethasone sodium $\mathrm{m}$-sulfobenzoate permeated through intact skin with donor with various periodical application of electric current at donor $\mathrm{pH}$ of 7.4

current.

3. The permeation flux of BA through the intact skin can be changed by switching electric current on and off during the course of experiments, which enables chronopharmacological control of ionic drug concentration in plasma.

4. Dexamethasone sodium m-sulfobenzoate (DMSB, MW=599) could also permeate through the intact skin by applying iontophoretic technique.

5. Computer simulation may be useful for prescribing better a TTS mode for iontophoretic treatment with a specific drug for a specific disease.

\section{Nomenclature}

$C_{D} \quad=$ concentration of solute of interest in donor

solution $\left[\mathrm{kg} / \mathrm{m}^{3}\right]$

D = apparent diffusion coefficient of solute of interest in

$K=$ partition coefficient defined by ratio of concentration at surface of the membrane to that in solution right adjacent to membrane $\left(\approx C_{D}\right) \quad[-]$

$L=$ thickness of a piece of membrane [m]

$Q_{t} \quad=$ cumulative amount of solute of interest permeated through membrane till time $t$ per unit membrane area

$\left[\mathrm{kg} / \mathrm{m}^{2}\right]$

$t \quad=$ experimental time [s] $\left(d Q_{t} / d t\right)_{S S}=$ steady state mass flux through skin $\quad\left[\mathrm{kgs}^{-1} \mathrm{~m}^{-2}\right]$

\section{Literature Cited}

Barrer, R. M.; Diffusion in and Through Solids, Cambridge University Press, England (1951)

Crank, J.; The Mathematics of Diffusion, 2-nd ed., p. 49-53, Clarendon Press, Oxford, England (1975)

Tojo, K., C. C. Chiang, Y. W. Chien; "Drug Permeation Across the Skin: Effect of penetrant hydrophilicity," J. Pharm. Sci., 76, 123126, 1987

Tojo, K.; "Mathematical Modeling of Transdermal Drug Delivery," J. Chem. Eng. Japan., 20, 300-308 (1987)

Tojo, K.; "Mathematical Model of Iontophoretic Transdermal Drug Delivery," J. Chem. Eng. Japan., 22, $512-518$ (1989)

Tojo, K., Y. W. Chien, C. C. Chiang, P. Keshary; "Effect of Stratum Corneum Stripping on Skin Penetration of Drug," J. Chem. Eng. Jpn., 21, 544-546 (1988) 